TTR

Traduction, terminologie, re?daction

\title{
Évolution de la recherche empirique sur l'interprétation de conférence
}

\section{Daniel Gile}

Volume 8, numéro 1, 1er semestre 1995

Orientations européennes en traductologie

URI : https://id.erudit.org/iderudit/037202ar

DOI : https://doi.org/10.7202/037202ar

Aller au sommaire du numéro

\section{Éditeur(s)}

Association canadienne de traductologie

\section{ISSN}

0835-8443 (imprimé)

1708-2188 (numérique)

Découvrir la revue

\section{Citer cet article}

Gile, D. (1995). Évolution de la recherche empirique sur l'interprétation de conférence. TTR, 8(1), 201-228. https://doi.org/10.7202/037202ar

\section{Résumé de l'article}

Évolution de la recherche empirique sur l'interprétation de conférence - Dans un historique de la recherche sur l'interprétation, les années 90 correspondent à une période de renouveau après une prise de conscience (années 50 et 60), une période expérimentale (années 65 à 75) et une période de stagnation due au protectionnisme de certains praticiens au pouvoir (75 à fin des années 80 ). Ce renouveau se traduit par une plus grande ouverture interdisciplinaire et inter-centres et par une sensibilisation à la recherche empirique, mais celle-ci reste faible : les chercheurs, surtout des interprètes-enseignants, sont peu nombreux et n'ont pas été formés à la recherche et aux disciplines cognitives pertinentes; les centres sont peu nombreux, petits, dispersés et sans infrastructure institutionnelle. Les perspectives d'évolution restent incertaines.
Tous droits réservés (C) TTR: traduction, terminologie, rédaction — Les auteurs, 1995
Ce document est protégé par la loi sur le droit d'auteur. L’utilisation des services d'Érudit (y compris la reproduction) est assujettie à sa politique d'utilisation que vous pouvez consulter en ligne. 


\section{Évolution de la recherche empirique sur l'interprétation de conférence}

\section{Daniel Gile}

\section{Introduction}

Par recherche empirique, nous entendons ici une recherche menée directement à partir de phénomènes d'interprétation observés sur le terrain, par opposition à la théorisation ou à l'analyse des observations ou des analyses d'autrui.

Nous distinguerons en outre, dans la recherche empirique, la recherche observationnelle (parfois appelée naturaliste) et la recherche expérimentale. La première part de données observées sur le terrain telles qu'elles se manifestent spontanément, sans intervention délibérée du chercheur; dans la seconde, une situation particulière, plus ou moins "contrôlée», est provoquée par le chercheur aux fins de sa recherche.

L'intérêt de la recherche empirique dans les disciplines comportementales est évident: à travers une observation systématique et de plus en plus fine, elle permet d'apporter des 
éléments susceptibles de renforcer ou d'affaiblir des hypothèses et théories bâties sur la base d'intuitions et d'observations personnelles recueillies sans mécanismes de vérification, notamment à l'égard des biais personnels, des phénomènes de non-représentativité des échantillons, des inexactitudes dans les évaluations quantitatives. En interprétation comme en traduction, la recherche empirique a une importance particulière, étant donné les aspirations des milieux professionnels à des applications, notamment en matière de qualité de la prestation et de formation.

La réflexion systématique sur l'interprétation a commencé vers les années 50, dans une période de «pré-recherche». Dans les années 60 , des psychologues et linguistes se sont penchés eux aussi sur l'interprétation, avec leurs propres méthodes d'investigation. Ce fut une première tentative scientifique, assez malheureuse. Vers le milieu des années 70 , les interprètes eux-mêmes prirent le relais, avec un retour à une introspection généralisatrice. Au cours de la deuxième moitié des années 80 se produisit un renversement des tendances, avec cette fois-ci une aspiration à une recherche plus scientifique (voir Gile, 1994, 1995). Le présent article retrace l'histoire de la recherche empirique sur l'interprétation depuis la période des premiers pas, en se concentrant sur les processus du mouvement de recherche plutôt que sur les contenus et résultats. Il débouche sur une analyse de la situation actuelle et des perspectives d'avenir.

\section{La période des premiers écrits}

Les premiers écrits sur l'interprétation de conférence furent rédigés par des praticiens, sur la base de leur expérience personnelle de la pratique et de l'enseignement de l'interprétation, avec des visées essentiellement didactiques. Durant cette période, on ne trouve pas de travaux de recherche proprement dits. Toutefois, les premiers écrits posent déjà des questions ou font des affirmations qui, dans une logique de démarche scientifique, appellent des vérifications et explorations empiriques. Ainsi, pour ne prendre que les idées les plus concrètes et les moins difficilement vérifiables (d'autres, 
portant sur des processus mentaux, appellent également des vérifications empiriques, mais celles-là sont plus difficiles à réaliser), on évoquera les affirmations de J. Herbert (1952) sur le vocabulaire «énorme» dont devrait disposer l'interprète (p. 5), sur l'impossibilité pour une même personne d'être bon traducteur et bon interprète à la fois (p. 6), sur l'efficacité de la répétition avec décalage ou shadowing dans la formation, ou les affirmations de $\mathrm{H}$. Van Hoof (1962) sur la supériorité de la consécutive par rapport à la simultanée en matière de précision. Ces questions restent d'actualité et suscitent encore bien des débats, notamment la question du shadowing (voir par exemple Kondo, 1992, et Lambert, 1992), mais n'ont fait l'objet d'aucune vérification empirique à ce jour. La carence en matière de travaux empiriques est un phénomène saillant dans la recherche sur l'interprétation. Il est d'ailleurs intéressant de noter à ce propos qu'un récent livre consacré à la recherche empirique en interprétation (Lambert et Moser-Mercer, 1994), dont les auteurs se proposaient d'y montrer la contribution de celle-ci à l'interprétation et à des disciplines connexes (p. 6), se compose en majorité d'articles déjà publiés ailleurs et se rapportant à des travaux datant de plusieurs années, voire de plus de vingt ans. Même si le livre en question ne décrit pas fidèlement la situation dans son ensemble (et notamment les travaux rapportés dans Gran et Taylor, 1990, et dans les différents numéros de la revue triestine The Interpreter's Newsletter parus depuis 1988), il reflète bien un paysage désertique en matière de recherche empirique. Cet état de fait est significatif à deux titres: d'une part, il explique la faiblesse de l'ensemble des résultats concrets obtenus jusqu'ici; d'autre part, il apparaît comme un intéressant marqueur clinique du mal sociologique dont souffre la communauté des chercheurs en interprétation.

\section{La période expérimentale}

C'est vers le milieu des années 60 que des scientifiques «extérieurs» (non interprètes) commencent à travailler sur l'interprétation. Les travaux des psychologues et linguistes, tels $\mathrm{P}$. Oléron et $\mathrm{H}$. Nanpon (1964), A. Treisman (1965), E. Lawson (1967), F. Goldman-Eisler 
(1967), H. Barik (1969) et D. Gerver (1971) ont été décrits maintes fois, quasiment dans toutes les thèses sur l'interprétation à l'exception de celles émanant de l'ESIT de Paris (dont les citations en matière d'interprétation sont plutôt endogames - voir notamment Brisset, 1993). Nous ne reviendrons donc pas sur leur contenu, et nous contenterons de faire quelques observations sur les caractéristiques du mouvement en tant que tel:

A. En dépit de son importance historique, la période expérimentale représente un mouvement de faible amplitude, avec une poignée de chercheurs isolés, par opposition à de véritables équipes ou «laboratoires». Leurs travaux sont ponctuels eux aussi, dans le temps comme dans leurs centres d'intérêt, à l'exception de ceux de D. Gerver, qui a cherché à cerner progressivement le phénomène de l'interprétation dans une série d'investigations assez variées portant notamment sur le débit du discours et ses conséquences, sur le bruit, sur les pauses, sur la compréhension et le rappel du discours par les interprètes (voir Gerver, 1976). Le mouvement des chercheurs non-interprètes a également été très limité dans le temps. Aucun d'eux ne continue à s'intéresser activement à l'interprétation (notons toutefois que la contribution de D. Gerver, importante et soutenue, a été interrompue par son décès). Nous pensons que les raisons de la courte durée de l'engagement des non-interprètes dans la recherche sur l'interprétation relèvent en grande partie de facteurs sociologiques (essentiellement l'exclusion des scientifiques par les praticiens en cours de conquête de territoire - voir Gile [1994, 1995]), plutôt que de la complexité de l'objet même de la recherche, parfois évoquée pour expliquer le faible état d'avancement dans ce domaine (Lambert et Moser-Mercer, 1994, p. 5).

B. La quasi-totalité des travaux de ces scientifiques, qui constituent la majorité des textes publiés pendant cette période, sont expérimentaux. Soulignons à ce propos qu'à l'exception de quelques interprètes des pays de l'Est, notamment G. Chernov en Union Soviétique, et de l'Autrichienne I. Pinter (Kurz), qui a soutenu en 1969 une thèse expérimentale en psychologie sur l'interprétation à 
l'université de Vienne, aucun praticien n'a effectué de recherche empirique pendant la même période.

C. Comme il a été dit et redit par différents auteurs interprètes (voir entre autres Stenzl, 1983, et Gile, 1995), la plupart des travaux de la période expérimentale présentent des faiblesses méthodologiques très importantes, qui remettent sérieusement en question leur validité en ce qui concerne les processus intervenant en interprétation de conférence. Ainsi, nombre de ces travaux ont pris comme sujets pour leurs expériences non pas des interprètes professionnels mais des élèves-interprètes, voire des «bilingues» sans aucune expérience ou formation en matière d'interprétation. Les tâches, matériaux et conditions expérimentales étaient eux aussi assez éloignés de l'interprétation, avec l' «interprétation» de phrases isolées, voire de mots isolés, l'interprétation dans des combinaisons linguistiques inhabituelles pour les sujets, l'évaluation de discours produits en fonction de critères propres à l'écrit et non pas à l'oral, ainsi que d'autres critères contestables, notamment en matière de définition des erreurs et omissions.

\section{La période des praticiens}

Vers le milieu des années 70 , les chercheurs non-interprètes avaient plus ou moins disparu de la scène de la recherche sur l'interprétation, alors que les praticiens prenaient la relève avec, entre 1975 et 1985, une masse de quelque 300 textes, y compris une dizaine de thèses de doctorat et plusieurs mémoires de M.A. (le tableau 1, fondé sur notre bibliographie personnelle, reflète probablement de manière assez fidèle la situation générale dans les pays occidentaux). Une fois de plus, dans cette masse, les travaux empiriques sont rares, une vingtaine environ, soit moins de $10 \%$ du total. Or, à la différence des écrits des années 50, qui étaient sans prétention, les textes des années 70 et 80 s'inscrivent en grande partie dans une optique universitaire et de recherche, et succèdent dans le temps aux travaux scientifiques des linguistes et psychologues de la période expérimentale. L'absence d'une recherche empirique de la part des praticiens revêt donc ici une signification plus profonde. 


$\begin{array}{llll}\text { Année } & \text { Auteur(s) } & \text { Type d'auteur } & \text { Type de texte } \\ 1975 & \text { Barik } & \text { Ext. } & \text { Article } \\ 1975 & \text { Seleskovitch } & \text { I., Ens. } & \text { Thèse } \\ 1975 & \text { Thiéry } & \text { I., Ens. } & \text { Thèse } \\ 1976 & \text { Gerver } & \text { Ext. } & \text { Article } \\ 1979 & \text { Anderson } & \text { I. } & \text { M.A. } \\ 1980 & \text { Goldman-Eisler } & \text { Ext. } & \text { Article } \\ 1982 & \text { Gustin } & \text { Étudiant } & \text { Mémoire } \\ 1983 & \text { Davies \& Cooper } & \text { Ext.? } & \text { Article } \\ 1983 & \text { Kurz } & \text { I., Ens. } & 2 \text { Articles } \\ 1983 & \text { Lambert, S. } & \text { Ext. } & \text { Thèse } \\ 1983 & \text { Mackintosh } & \text { I., Ens. } & \text { M.A. } \\ 1983 & \text { Meak } & \text { I., Ens. } & \text { Monographie } \\ 1983 & \text { Salevsky } & \text { I., Ens.? } & \text { Thèse } \\ 1984 & \text { Bühler } & \text { Ext. } & \text { Article } \\ 1984 & \text { Gerver } \text { et } \text { al. } & \text { Ext., I., Ens. } & \text { Article } \\ 1984 & \text { Gile } & \text { I., Ens. } & \text { Thèse } \\ 1984 & \text { Gile } & \text { I., Ens. } & \text { Article } \\ 1984 & \text { Kurz \& Kolmer } & \text { I., Ens. } & \text { Article } \\ 1985 & \text { Gile } & \text { I., Ens. } & \text { Article } \\ 1985 & \text { Lambert/Lambert } & \text { Ext. } & \text { Article }\end{array}$

Abréviations:

I.: Interprète

Ens.: Enseignant en interprétation

Ext.: Chercheur non-interprète

Tableau 1: Tableau synoptique des travaux empiriques (ou comprenant une partie empirique) sur l'interprétation dans les pays occidentaux entre 1975 et 1985.

Les travaux empiriques des praticiens durant cette période (par opposition à ceux des chercheurs «extérieurs») présentent par ailleurs quelques caractéristiques propres:

A. Ils ne font généralement pas appel à des concepts et modèles scientifiques relevant de la psychologie et de la linguistique, et se 
cantonnent dans le vécu quotidien de l'interprète. Ainsi, D. Seleskovitch (1975) étudie concrètement les notes que prennent les interprètes en consécutive, C. Thiéry (1975) interroge les interprètes classés «bilingues» à l'AIIC (Association Internationale des Interprètes de Conférence) sur leur expérience linguistique personnelle, L. Anderson (1979) compare la qualité de la prestation de l'interprète sous différentes conditions de travail, L. Meak (1983) interroge les médecins sur la qualité de l'interprétation. On notera quelques exceptions, telle J. Mackintosh (1983), qui évoque les idées de Kintsch et Van Dijk sur la compréhension du discours, ainsi que D. Gile (1984), qui se sert de quelques concepts relevant de la psychologie cognitive et de la théorie mathématique de l'information pour analyser les difficultés de la simultanée. Il n'en demeure pas moins que dans ces deux derniers cas, ces concepts ne sous-tendent pas la démarche empirique proprement dite. En effet, tant l'examen des discours interprétés avec relais dans l'étude de $\mathbf{J}$. Mackintosh que la vérification de la difficulté que présentent les noms propres à l'interprète dans l'étude de D. Gile, partent de préoccupations d'ordre professionnel et ne reposent pas véritablement sur les concepts théoriques évoqués dans les textes respectifs. Par contraste, rappelons que la plupart des travaux des psychologues et linguistes partaient d'une idée ou d'un concept théorique et avaient pour fonction de les vérifier.

B. Les travaux des praticiens sont très simples sur le plan méthodologique, notamment dans leur traitement statistique, plus souvent descriptif qu'inférentiel (à l'exception toutefois des travaux de L. Anderson et de J. Mackintosh, réalisés dans des universités sous la direction de chercheurs apparemment habitués à des exigences inférentielles en la matière). Ainsi, la thèse de D. Seleskovitch (1975) repose sur une seule expérience dans laquelle elle a demandé à 12 interprètes d'interpréter deux discours en consécutive, sans aucun montage expérimental. L'expérience de D. Gile sur les noms propres (1984) suit un même schéma, le contrôle s'exerçant uniquement sur la variable «discours de départ». D'autres travaux empiriques de praticiens sont de simples enquêtes par questionnaire et interviews (Thiéry, 1975; Meak, 1983) sous leur forme la plus rudimentaire. 
C. Du fait de leur qualité d'interprètes, les praticiens évitent les problèmes de validité qu'ils reprochent aux travaux des psychologues et linguistes. Leurs sujets sont bien des interprètes, et dans les études expérimentales, les conditions sont assez proches des conditions de travail sur le terrain. En revanche, ils pêchent parfois dans le processus inférentiel, qui manque de rigueur. Ainsi, C. Thiéry (1975) pose comme base de la définition du «bilinguisme vrai» la perception du locuteur par les communautés linguistiques concernées, sans vérifier si ce critère remplit des conditions minimum de mesurabilité d'abord, et de sensibilité, de sélectivité et de stabilité ensuite. Ainsi, D. Seleskovitch (1975) pense avoir démontré le bien-fondé de son hypothèse de "déverbalisation» (la non-intervention d'éléments linguistiques du discours en langue de départ dans la préparation du discours correspondant en langue d'arrivée) par le seul fait que les interprètes prennent des notes différentes lors de l'écoute d'un même' discours en langue de départ, sans essayer de chercher une autre explication à cette variabilité.

D. De même que les travaux empiriques des psychologues et linguistes de la période expérimentale, ceux des praticiens ne présentent pour ainsi dire pas de suivi (un ou deux travaux empiriques sur le même sujet par chercheur). Toutefois, chez les scientifiques, la cause du phénomène peut être attribuée au petit nombre de chercheurs isolés intéressés par le domaine; chez les interprètes, ce sont probablement des questions de disponibilité et de motivation qui sont en cause, comme il est expliqué plus loin. On constate d'ailleurs que parmi les travaux empiriques des praticiens durant cette période (une douzaine), plus de la moitié sont des thèses, mémoires de M.A. ou mémoires de fin d'études. Si une partie non négligeable de leurs auteurs ont continué à écrire des textes sur l'interprétation (en particulier D. Seleskovitch), deux seulement, I. Kurz et D. Gile, ont continué dans la voie de la recherche empirique. Le même phénomène se retrouve d'ailleurs dans la période actuelle de "renouveau», avec un nombre non négligeable de mémoires de fin d'études qui restent sans suivi.

E. D'autre part, les travaux empiriques des interprètes ne sont pas constitués en édifices où chaque chercheur apporte sa contribution 
à l'ouvrage, comme c'est généralement le cas dans les disciplines bien établies. Ils contrastent par là fortement avec les travaux non empiriques de la même période, qui font preuve d'une grande cohésion, notamment autour de sujets tels que l'enseignement de l'interprétation.

F. Phénomène qui n'a rien de surprenant mais qui apparaît avec une grande netteté à travers les chiffres, la quasi-totalité des travaux empiriques sur l'interprétation sont réalisés par des enseignants de l'interprétation. Les auteurs enseignants sont également fortement majoritaires dans les autres types de textes sur l'interprétation, mais dans ceux-ci, notamment les textes factuels sur les conditions de travail ou sur la pratique du métier, on trouve également un nombre non négligeable d'interprètes non-enseignants, ce qui n'est pas le cas dans la recherche empirique.

\section{La période de renouveau}

La domination d'un dogme et les stratégies de fermeture de ses tenants ayant fortement freiné l'évolution de la pensée (voir Gile, 1995), une certaine stagnation dans la recherche en interprétation finit par devenir évidente. En réaction, des praticiens se tournèrent une fois de plus vers une démarche plus ouverte pour faire progresser la recherche. Une conférence sur l'enseignement de l'interprétation organisée par l'école de traduction et d'interprétation de l'université de Trieste en 1986 (voir Gran et Dodds, 1989) constitue probablement un bon point de repère pour marquer le démarrage de la période de renouveau qui s'ensuivit.

Ce renouveau se démarque de la période des praticiens surtout par des attitudes, que l'on pourrait caractériser principalement comme plus ouvertes:

A. Sur le plan sociologique, on notera essentiellement une tendance au décloisonnement et à la déhiérarchisation entre les écoles. Ainsi, dès le colloque de Trieste, les activités de réflexion, de recherche et de publication sont plus largement réparties sur le plan géographique, avec une activité significative non seulement dans les 
quelques grands centres à Paris, à Genève, à Leipzig et à Moscou, mais aussi dans des écoles et centres universitaires très peu connus jusqu'alors, notamment en Italie, en Finlande, au Japon et en Australie. Cette évolution s'explique essentiellement par deux facteurs: d'une part, l'arrivée d'une nouvelle génération de chercheurs, qui rejette la chape trop rigide du dogme issu de la période des praticiens et expérimente avec de nouvelles idées, et d'autre part la multiplication des contacts nationaux et internationaux grâce à des conférences et colloques, livres, revues et autres vecteurs d'information toujours plus nombreux et faciles d'accès (la revue triestine The Interpreter's Newsletter est gratuite, comme l'est le Bulletin de l'IRTIN, qui est lu actuellement dans une quarantaine de pays à travers le monde).

B. Sur le plan idéologique, cette même recherche de renouvellement conduit à l'ouverture à de nouvelles idées, parfois contraires à celles héritées de la période des praticiens (notamment en matière de formation à l'interprétation, de spécificité de l'interprétation par rapport aux langues), mais aussi à de nouvelles disciplines, telles que la psychologie cognitive, la linguistique et la neurolinguistique, et à de nouvelles méthodes, notamment expérimentales et quantitatives, souvent condamnées durant la période des praticiens.

Pourtant, si ces nouvelles attitudes sont associées à un déplacement tant qualitatif que quantitatif de la productivité dans la recherche par rapport à la période précédente, en ce qui concerne la recherche empirique, des contraintes importantes continuent à peser lourdement sur la communauté des chercheurs en interprétation. Cette deuxième partie du présent article analyse la situation au regard de ces contraintes.

\subsection{Nombre et importance relative des textes empiriques}

L'analyse ci-dessous se fonde sur notre bibliographie personnelle, qui est probablement assez complète pour les pays occidentaux et le Japon et qui couvre probablement les principales entrées publiées dans d'autres pays du monde. Elle résulte en effet du dépouillement systématique des principales revues de traduction et d'interprétation, 
ainsi que des informations circulant à l'intérieur de l'IRTIN, réseau international d'information sur la recherche en interprétation. D'après cette bibliographie, entre 1986 et 1994, quelque 635 textes de recherche ont été publiés de par le monde. Parmi eux, seuls 97, soit quelque $15 \%$, rapportent des études relevant de la recherche empirique. Qui plus est, une partie non négligeable de ces textes rapportent les mêmes études (articles publiés deux fois, mémoires ou thèses résumés par la suite dans un article, livres collectifs reprenant des articles, articles passant en revue une série de résultats présentés précédemment). Tant en proportion qu'en chiffres absolus, la composante empirique dans la recherche active sur l'interprétation reste donc très faible.

\subsection{Les auteurs de textes empiriques}

Dans le même ordre d'idées, le nombre d'auteurs de textes rapportant des travaux empiriques (ci-après 'textes empiriques') est a priori relativement important, puisqu'il est de 61 personnes. Toutefois, l'image se précise de manière moins encourageante quand on recherche les auteurs ayant une activité de recherche suivie, par opposition à des auteurs ayant réalisé une seule étude, dans le cadre de la préparation d'un diplôme par exemple, pour s'éloigner de la recherche par la suite. Ainsi, le nombre d'auteurs ayant publié au moins trois textes empiriques n'est que de 11 personnes, et seuls cinq auteurs (L. Gran et F. Fabbro de Trieste, S. Lambert d'Ottawa, I. Kurz de Vienne et D. Gile de Paris) ont publié au moins 4 textes empiriques chacun. En fait, la production de ces cinq auteurs représente à elle seule 26 entrées, soit près de $27 \%$ du total des textes. Dès lors, il semble difficile de parler de l'existence d'une vraie communauté de chercheurs empiriques actifs dans le domaine de l'interprétation, même si l'on admet que quelques études empiriques sont en cours et n'ont simplement pas encore donné lieu à des publications.

\subsection{Répartition géographique des travaux empiriques}

Si l'on examine la répartition des études empiriques par pays de provenance, on constate une très forte prédominance de l'Italie, et 
plus précisément de la Scuola Superiore di Lingue Moderne per Interpreti e Traduttori de la Universita degli Studi di Trieste, avec 33 entrées sur 97, soit plus du tiers du total. L'école de Trieste est suivie, à très grande distance, par l'Autriche, le Canada, la Finlande et le Japon, chacun avec 9 entrées, puis par les Etats-Unis et la France, chacun avec 6 entrées. L'Australie apporte 5 entrées, et les autres pays une, deux ou trois entrées chacun (tableau 2):

$\begin{array}{lll}\text { Pays } & \text { Nbre de textes empiriques } & \text { \% du total } \\ \text { Italie } & 33 & \text { env. } 34 \% \\ \text { Autriche } & 9 & \text { env. } 9,3 \% \\ \text { Canada } & 9 & \text { env. } 9,3 \% \\ \text { Finlande } & 9 & \text { env. } 9,3 \% \\ \text { Japon } & 9 & \text { env. } 9,3 \% \\ \text { États-Unis } & 6 & \text { env. } 6,2 \% \\ \text { France } & 6 & \text { env. } 6,2 \% \\ \text { Australie } & 5 & \text { env. } 5,1 \% \\ & & \text { env. } 88,7 \%\end{array}$

Tableau 2: Répartition des textes empiriques (1986-1994) par pays

Cette répartition s'explique essentiellement par des facteurs institutionnels, qui ont aussi une incidence motivationnelle. On notera en effet qu'en Italie et en Finlande, une grande partie des travaux empiriques sont des projets de fin d'études nécessaires à l'obtention du diplôme. Les étudiants ont l'obligation de réaliser un projet, et dans la mesure où tant en Italie qu'en Finlande, les enseignants sont très tournés vers la démarche empirique, la proportion des mémoires qui suivent celle-ci est élevée. On remarquera à ce propos que le nombre de travaux empiriques dans les autres écoles où des mémoires de fin d'études sont requis (notamment Heidelberg et Vienne) est bien plus faible, les étudiants préférant souvent consacrer leurs efforts à des mémoires 
terminologiques. On s'intéressera aussi à la production australienne, que l'on attribuera sans difficulté à la présence dynamique pendant quelques années à l'université du Queensland d'une jeune linguiste formée à la recherche empirique et recrutée à un poste de chercheur en traduction et en interprétation. Depuis, ce poste a disparu, et l'activité australienne s'est éteinte.

\subsection{Sujets traités}

Les sujets traités dans les travaux empiriques durant la période de renouveau présentent une certaine variété, mais peuvent être classés pour la plupart en quatre grands «blocs», aux fins de l'analyse:

A. Les travaux traitant de modalités ou conditions de travail de l'interprétation ne faisant pas appel à des connaissances relevant de domaines connexes.

Cette catégorie nous semble importante dans la mesure où elle montre certaines possibilités de recherche empirique en interprétation accessibles à des interprètes n'ayant pas de connaissances en linguistique, en psychologie, en psycholinguistique ou en neurophysiologie et ne disposant pas d'équipements de laboratoire spécialisés.

Dans ce groupe, on dénombre plus de trente travaux, soit un tiers environ de l'ensemble des travaux empiriques réalisés (le décompte n'est pas exact, car nous n'avons pas accès à l'ensemble des textes et ne pouvons classer certains que d'après leur titre; par ailleurs, il existe de nombreux travaux qui ont fait l'objet de deux, voire trois publications distinctes, ce qui fait que le nombre de textes ne correspond pas exactement au nombre de travaux réalisés). Parmi les sujets abordés figurent l'effet du contexte et du contact visuel avec l'orateur sur la performance de l'interprète (Balzani, 1990), l'interprétation des nombres (Alessandrini, 1990; Braun, 1992; Crevatin, 1991), la qualité du travail (une douzaine de travaux), le travail lexicologique et terminologique (Gile, 1989; Moser-Mercer, 1992), des comparaisons entre simultanée et traduction à vue (Viezzi, 1990) et entre simultanée et traduction 
(Viezzi, 1993), ainsi que la spécialisation de l'interprète (Schweda-Nicholson, 1986, 1989).

Ces travaux, observationnels ou expérimentaux, marquent un progrès par rapport aux textes introspecto-normatifs de la période des praticiens par une volonté manifeste de confirmer des hypothèses, notamment par la quantification. Les montages et méthodes restent néanmoins simples dans la grande majorité des cas.

B. Les travaux sur des facteurs physiologiques et neurophysiologiques.

Ceux-ci, au nombre d'une petite vingtaine, portent notamment sur la latéralisation cérébrale chez les interprètes (une dizaine), ainsi que sur l'activité cérébrale en interprétation (Petsche, 1993; Kurz, 1994), sur l'activité cardiaque (Klonowicz, 1994), sur la réaction de la pupille à la charge mentale (Tommola et Niemi, 1986; Tommola et Hyönä, 1990), sur les fréquences vocales (Daro, 1990).

Il s'agit des travaux les plus complexes à réaliser, en ce sens qu'ils demandent des connaissances spécialisées ainsi que des appareils de laboratoire. Ils sont pour la plupart effectués en équipe par des spécialistes (neurophysiologues, ophtalmologistes, etc.) ou des équipes mixtes interprètes/spécialistes. On notera la grande proportion de travaux réalisés dans ce domaine à Trieste, sans aucun doute grâce aux liens que l'école d'interprétation a su tisser avec les neurophysiologues, notamment F. Fabbro.

C. Des travaux relevant de la psycholinguistique et de la psychologie cognitive.

Il s'agit d'une petite dizaine de travaux (ayant donné lieu à des textes multiples) sur la compréhension (Dillinger, 1989), sur la mémoire (De Felice, 1992), sur la déverbalisation (Isham, 1994), sur la segmentation du discours à l'écoute (Davidson, 1992) et sur les pauses et hésitations (Ovaska, 1987). Réalisés pour plus de la moitié par des non-interprètes, ils font parfois appel à des 
connaissances spécialisées approfondies, et à des méthodes expérimentales assez élaborées, mais sans appareillage compliqué. Ils sont toutefois difficiles d'accès aux praticiens.

D. Des travaux relevant de la linguistique appliquée.

Là aussi, on dénombre une dizaine de travaux, réalisés en partie par des praticiens et en partie par des linguistes: sur les transformations syntaxiques intervenant dans l'interprétation (Giambagli, 1990), les niveaux de politesse ( $\mathrm{Ng} \&$ Obana, 1992), les changements de registre (Shlesinger, 1989), des caractéristiques spécifiques de certaines langues (Gile, 1992), les erreurs et maladresses linguistiques des élèves-interprètes(Gile, 1987). Ces travaux font en partie appel à des connaissances linguistiques spécialisées (c'est notamment le cas du travail de M.A. de M. Shlesinger, 1989, dans lequel l'auteur montre que l'interprétation de discours présentant les caractéristiques linguistiques de l'écrit tend à produire des discours ayant des caractéristiques plus proche de l'oral et inversement), mais se font également avec des outils descriptifs non spécialisés, dont l'accès est donc aisé aux praticiens.

\section{Synthèse, problèmes et perspectives}

\subsection{Le renouveau}

Outre l'ouverture d'esprit, les éléments qui permettent de qualifier la dernière période de «renouveau» dans le sens de la recherche empirique sont donc les suivants:

A. L'implantation d'une activité empirique soutenue en de nouveaux lieux, notamment un centre, à savoir l'école de traduction et d'interprétation de l'université de Trieste, et une région géographique, à savoir la Finlande. On notera d'ailleurs un déplacement "géographique» important du centre de gravité de l'activité interprétative: parmi les huit pays les plus producteurs en matière de recherche empirique (voir tableau 2), six sont de nouveaux venus parmi les grands. Seules l'Autriche et la France avaient déjà une importance significative auparavant. Le rang de 
l'Autriche était attribuable à la productivité soutenue d'Ingrid Kurz, qui se poursuit. Celui de la France tournait autour des activités de l'ESIT pendant la période des praticiens. La grande perte de poids de l'ESIT dans la masse des textes sur l'interprétation constitue peut-être, avec la montée de l'école de Trieste, le changement le plus important dans le paysage institutionnel de la recherche.

B. Le développement d'une activité suivie avec des réplications autour de quelques thèmes tels que la latéralisation cérébrale chez les interprètes, la perception de la qualité du travail, l'importance du contact visuel de l'interprète avec le délégué, l'interprétation des nombres, etc. est une autre nouveauté. Cette activité suivie, bien qu'encore peu importante, contraste fortement avec le tableau que présentaient les travaux empiriques isolés de la période des praticiens.

C. Pendant cette période de renouveau sont également apparus quelques projets de collaboration interdisciplinaire, notamment sur le plan physiologique (Tommola et Niemi, Tommola et Hyönä) et sur le plan neurophysiologique (Gran et Fabbro, Daro et Fabbro, Kurz et Petsche). On notera une nouvelle fois que les chercheurs 'extérieurs' qui s'intéressent à l'interprétation ne sont plus ceux de la période expérimentale.

\subsection{Les raisons de la faiblesse de la recherche empirique}

Il n'en demeure pas moins que, en dépit de nombreux appels lancés depuis toujours en faveur de la recherche empirique (voir notamment Anderson, 1979; Stenzl, 1983; Shlesinger, 1989), comme il est indiqué plus haut, quantitativement parlant, celle-ci reste faible, ce qui explique aussi l'absence pour l'instant de réalisations solides. Il y a bien des résultats ponctuels, mais que l'on a du mal à réunir en un tout qui réduirait significativement le flou que présente actuellement l'image de l'entité «interprétation» sur le plan psychologique ou linguistique. Nous rejoignons en ce sens les préoccupations de F. Pöchhacker, pour qui 
The picture presented by recent interpretation research rather resembles isolated pieces and patches of a jigsaw puzzle with little indication as to their relative position and the limits or frame of the image to be construed. (1993, p. 52)

Il nous semble toutefois que cette situation résulte non pas d'une absence de cadre général, comme le pense $F$. Pöchhacker, mais de l'insuffisance des travaux empiriques. En tout état de cause, il apparaît intéressant de tenter d'expliquer cette faiblesse. En cours d'analyse, on voit d'ailleurs apparaître des facteurs qui portent également sur la faiblesse de la recherche en général, au-delà de la recherche empirique.

Une première explication de l'état de développement peu avancé de la recherche empirique en interprétation est d'ordre historique. Si l'on considère que l'interprétation appartient au domaine de la traduction, comme le pensent de nombreux chercheurs (voir Stenzl, 1983, mais aussi de plus récents textes de H. Salevsky de Berlin, 1993, et de F. Pöchhacker de Vienne, 1993), elle se rattache à une longue tradition qui s'est située dans le champ des belles-lettres d'une part et de la philosophie de l'autre. On notera d'ailleurs que la recherche empirique en traduction est tout aussi récente et peu développée qu'en interprétation, voire moins développée encore. $\mathrm{La}$ philosophie «descriptive» de la «Manipulation School» se rattache à la démarche empirique (et a notamment donné naissance à quelques travaux malheureusement peu connus de l'université de Louvain). On relèvera aussi quelques travaux autour de la méthode des TAP ou «Think-Aloud Protocols», notamment en Allemagne, au Canada et en Finlande (voir par exemple Tirkkonen-Condit, 1991; Tirkkonen-Condit et Laffling, 1993), mais les travaux dans ce domaine semblent marquer le pas.

On comprendra sans peine que les notions de fidélité et de traduisibilité, qui ont préoccupé les auteurs et les lecteurs des traductions depuis l'antiquité, ont été à l'origine d'une réflexion non empirique. En effet, en traduction, l'attention s'est toujours portée sur le produit de l'opération, à savoir le texte. En revanche, en interprétation s'est posée dès le départ, et avec beaucoup d'acuité, 
la question des performances, donc du processus (ce qui apparaît très nettement dans les écrits de J. Herbert, de J.-F. Rozan, de G. Ilg, de E. Paneth et de D. Seleskovitch au cours des années 50 et $60)$. Il aurait donc semblé naturel qu'en interprétation, la recherche s'oriente vers l'investigation empirique, ce qui n'a pas été le cas. L'explication doit être cherchée ailleurs.

En examinant la cristallisation d'une première activité de réflexion et de recherche sur l'interprétation dans les années 50, 60 et 70 , on remarquera deux sortes d'auteurs parmi les praticiens:

- Des auteurs de textes pratiques visant des fins pratiques, sans prétentions universitaires (c'est notamment le cas de J. Herbert, de J.-F. Rozan et de G. Ilg de Genève, ainsi que de P. Longley de Londres).

- Des auteurs qui se placent d'emblée dans une perspective universitaire. Parmi ceux-ci, on notera plus particulièrement D. Seleskovitch, qui mit en place dans les années 70 un programme doctoral en interprétation et traduction à l'ESIT (Paris). Cette réussite universitaire fut à l'origine d'un important mouvement auquel se rallièrent non seulement des praticiens exerçant à Paris (notamment M. Lederer et K. Déjean Le Féal), mais aussi des interprètes enseignant dans d'autres écoles, dispersées à travers le monde, qui profitaient ainsi de ce statut universitaire nouvellement acquis de l'interprétation. Or, la démarche de D. Seleskovitch était résolument anti-expérimentale, et les praticiens qui gravitaient autour d'elle ont suivi la même voie de la théorisation fondée sur l'expérience personnelle. À l'exception de la thèse de C. Thiéry de l'ESIT (1975), les travaux empiriques réalisés pendant toute cette période, notamment par I. Pinter (Kurz) (1969) et L. Anderson (1979) ainsi que les idées et travaux des psychologues et linguistes sur l'interprétation n'ont jamais été cités par D. Seleskovitch et son groupe. Et pourtant, ce n'est pas par ignorance, puisque plusieurs de ces chercheurs ont participé au colloque organisé par de tels chercheurs extérieurs à Venise en 1977, précisément dans le but de mettre en place une recherche collaborative avec les interprètes praticiens (voir Gerver et Sinaiko, 1978). L'attitude défensive, et parfois agressive du groupe des interprètes vis-à-vis des chercheurs extérieurs s'explique mal par les seules erreurs de ces derniers 
évoquées plus haut. Après tout, loin d'afficher une attitude de mépris ou d'arrogance face aux praticiens, dont les travaux manquaient manifestement de rigueur scientifique, ils leur ont plutôt témoigné un intérêt amical, qui transparaît bien dans les textes de la conférence de Venise (Gerver et Sinaiko, 1978). Leur rejet par les praticiens semble mieux s'expliquer par deux autres éléments, qui ne sont d'ailleurs pas indépendants l'un de l'autre: d'une part, le désir de préserver un territoire universitaire nouvellement conquis, et d'autre part, la très probable insuffisance des compétences des interprètes en matière de recherche (il semblerait qu'aucun parmi eux n'ait été formé à la recherche, ni d'ailleurs à la psychologie cognitive, à la psycholinguistique ou à la neurolinguistique). On notera sans surprise que les interprètes favorables dès cette époque à la recherche interdisciplinaire (notamment I. Kurz et B. Moser) avaient, elles, bénéficié d'une telle formation.

Il est également intéressant de noter à ce propos qu'à l'heure actuelle, quelque quinze ans plus tard, les tenants d'une démarche plus proche de la démarche scientifique, préférentiellement axée sur des explorations empiriques et ouverte sur d'autres disciplines, se retrouvent surtout parmi une "nouvelle» génération qui n'a pas de «territoire» ou de statut à défendre (mais l'on rétorquera peut-être qu'elle a un territoire à conquérir).

Toutefois, ces nouveaux chercheurs doivent eux aussi surmonter des obstacles. Le premier est le même que celui de leurs prédécesseurs; il s'agit de la double absence de formation, dans les méthodes de recherche d'une part, et dans les domaines pertinents (linguistique, psychologie, etc.) de l'autre. Le second est l'absence d'une infrastructure administrative, matérielle et humaine pour leur recherche. À l'exception de Trieste, et notamment de la précieuse présence engagée du neurophysiologue $F$. Fabbro, aucun centre n'offre de soutien financier, scientifique et logistique significatif aux candidats à la recherche sur l'interprétation. Face à de telles contraintes, il faut être particulièrement motivé pour échapper à la tentation de l'introspection et pour s'engager dans la voie ardue de la recherche empirique, au prix d'un temps et de pertes financières assez considérables. Dès lors, on ne s'étonnera pas du petit nombre 
de travaux empiriques finalement réalisés en interprétation, avec un très petit nombre d'interprètes.

\subsection{Perspectives}

Étant donné les besoins de l'interprétation en termes de performance, il nous semble inévitable qu'à terme, celle-ci sera considérée comme tombant naturellement dans le champ de compétence des disciplines comportementales, ce qui n'est pas nécessairement le cas de la traduction écrite, et surtout de la traduction littéraire. Il nous semble également naturel de considérer que la recherche sur l'interprétation en tant que discipline comportementale ne saurait avancer sans une importante composante empirique. Le tournant amorcé depuis la deuxième moitié des années 80 ne nous semble donc guère surprenant. Les conditions restent pourtant peu favorables à un développement d'une telle recherche; du côté des praticiens, les connaissances et le savoir-faire scientifique, ainsi que la disponibilité et la motivation, restent très insuffisants, sans qu'un élément extérieur vienne les renforcer. Du côté des scientifiques, et notamment des psychologues et linguistes, les aides institutionnelles restent très limitées (infrastructure, postes de chercheurs, financement), contrairement aux aides à la traduction automatique. La chose est probablement due en partie au faible enjeu économique que représente l'interprétation dans le monde (à l'exception du cadre que constituent quelques organisations telles que la Commission de l'Union européenne); mais peut-être s'explique-t-elle aussi par le fait que lors de la période des praticiens, l'on a occulté les problèmes réels de performance et de formation en présentant de l'interprétation une image idéalisée où l'interprète compétent travaillant dans des conditions adéquates accomplit sa mission sans difficulté, créant par là l'illusion que la recherche n'est ni nécessaire, ni utile.

\section{Conclusion}

Le tournant actuel annonce-t-il une modification de fond de la situation? Peut-être, dans la mesure où il ouvre l'interprétation à la 
communauté scientifique des sciences cognitives, et donc à un développement naturel. Du côté des interprètes praticiens, essentiellement des étudiants et enseignants de l'interprétation, si l'exemple de Trieste est suivi dans d'autres centres, une certaine progression devrait également être possible. Nous proposons d'ailleurs (Gile, 1995) un certain nombre d'actions en faveur de la formation, de la collaboration et de la motivation, mais sommes conscient de leur dépendance vis-à-vis d'une véritable volonté politique dans les écoles de traduction et d'interprétation. En l'absence d'une telle volonté et de transformations institutionnelles importantes en faveur de la recherche, il faut s'attendre à une avance très lente, au moins dans les premières années. En même temps se pose la question de la destinée de l'interprétation même, dont certains prédisent la mort dans un avenir proche, avec la généralisation de l'apprentissage des langues étrangères et l'internationalisation de l'anglais. $\mathrm{Si}$ cette prophétie devait se réaliser, la recherche en la matière n'aurait plus d'objet. En revanche, les tentatives de mise au point de systèmes de reconnaissance automatique de la parole chaînée, puis de systèmes automatiques d'interprétation simultanée, pourraient redonner vigueur à la recherche empirique sur l'interprétation, avec une forte composante linguistique.

En tout état de cause, pour l'historien des sciences comme pour le sociologue des sciences, le cas de la recherche en interprétation semble particulièrement intéressant.

Daniel Gile: ISIT, 21 rue d'Assas, 75270 Paris Cedex, France

\section{Références}

ALESSANDRINI, Maria Serena (1990). «Translating Numbers in Consecutive Interpretation: An Experimental Study), The Interpreter's Newsletter 3, pp. 77-80. 
ANDERSON, Linda (1979). Simultaneous Interpretation: Contextual and Translation Aspects, M.A. thesis, Concordia University, Montréal, non publié.

BALZANI, Maurizio (1990). "Le contact visuel en interprétation simultanée: résultats d'une expérience (français-italien)», in Gran et Taylor, dir. (1990), pp. 93-100.

BARIK, Henry C. (1969). A Study of Simultaneous Interpretation. Thèse de doctorat, University of North Carolina, non publiée.

BRAUN, S. (1992). Analisi Neurolinguistica degli errori nei numerali durante l'interpretazione simultanea: un studio sperimentale. Mémoire de fin d'études, Scuola Superiore di lingue moderne per Interpreti e Traduttori, Universita degli Studi di Trieste, non publié.

BRISSET, Annie (1993). Compte rendu de Lederer Marianne, réd., 1990, «Études traductologiques», Target V(2), pp. 255-258.

CREVATIN, Alessandra (1991). La traduzione dei numeri in interpretazione simultanea: un contributo sperimentale. Mémoire de fin d'études, Scuola Superiore di lingue moderne per Interpreti e Traduttori, Universita degli Studi di Trieste, non publié.

DARO, Valeria (1990). "Voice Frequency and Simultaneous Interpretation», The Interpreter's Newsletter 3, pp. 88-92.

DAVIDSON, Peter (1992). "Segmentation of Japanese Source Language Discourse in Simultaneous Interpretation», The Interpreter's Newsletter, Special Issue 1, pp. 2-11.

DE FELICE, A. (1992). Memoria a breve termine $e$ interpretazione simultanea: un contributo sperimentale. Mémoire de fin d'études, Scuola Superiore di lingue moderne per Interpreti e Traduttori, Universita degli Studi di Trieste, non publié. 
DILLINGER, Mike (1989). Component Processes of Simultaneous Interpreting. Thèse de doctorat, Department of Educational Psychology, McGill University, Montréal, non publiée.

GERVER, David (1971). Simultaneous Interpretation and Human Information Processing. Thèse de doctorat, Université d'Oxford, non publiée.

(1976). «Empirical Studies of Simultaneous Interpretation: a Review and a Model», in Brislin R., dir. (1976). Translation, New York, Gardner Press, pp. 165-207.

GERVER, David \& H. Wallace SINAIKO, dir. (1978). Language Interpretation and Communication. New York and London, Plenum Press.

GIAMEAGLI, Anna (1990). "Transformations grammaticales, syntaxiques et structurales dans l'interprétation consécutive vers l'italien d'une langue latine et d'une langue germanique», The Interpreter's Newsletter 3, pp. 98-111.

GILE, Daniel (1987). «Les exercices d'interprétation et la dégradation du français: une étude de cas», Meta XXXII(4), pp. 420-428.

(1992). «Predictable Sentence Endings in Japanese and Conference Interpretation», The Interpreter's Newsletter, Special Issue 1, pp. 12-23.

(1994). "Opening up in Interpretation Studies», in Snell-Hornby, Pöchhacker et Kaindl, dir. (1994), pp. 149-158.

(1995). Regards sur la recherche en interprétation de conférence. Lille, Presses universitaires de Lille.

GOLDMAN-EISLER, Frieda (1967). «Sequential Temporal Patterns and Cognitive Processes in Speech», Language and Speech 10, pp. $122-132$. 
GRAN, Laura et John DODDS, dir. (1989). The Theoretical and Practical Aspects of Teaching Conference Interpretation. Udine, Campanotto Editore.

GRAN, Laura et Christopher TAYLOR, dir. (1990). Aspects of Applied and Experimental Research on Conference Interpretation. Udine, Campanotto Editore.

HERBERT, Jean (1952). Manuel de l'interprète. Georg, Genève.

ISHAM, William P. (1994).«Memory for Sentence Form after Simultaneous Interpretation: Evidence Both For and Against Deverbalization", in Lambert et Moser-Mercer, dir. (1994), pp. 191-211.

KLONOWICZ, Tatiana (1994). «Putting one's Heart in Simultaneous Interpretation», in Lambert et Moser-Mercer, dir. (1994), pp. 213-224.

KONDO, Masaomi (1992). "The Effectiveness of Shadowing - a European Debate» (en japonais), Interpreting Research 2, pp. 43-51.

KURZ, Ingrid (1994). "A Look into the Black Box: EEG Probability Mapping during Mental Simultaneous Interpreting), in Snell-Hornby, Pöchhacker et Kaindl, dir. (1994), pp. 199-207.

LAMBERT, Sylvie (1992). «Shadowing», Meta XXXVII(2).

LAMBERT, Sylvie et Barbara MOSER-MERCER, dir., (1994). Bridging the Gap. Empirical Research in Simultaneous Interpretation. Amsterdam/Philadelphia, John Benjamins.

LAWSON, E.A. (1967). «Attention and Simultaneous Translation», Language and Speech 10, pp. 29-35.

MACKINTOSH, Jennifer (1983). Relay Interpretation: An Exploratory Study. M.A. Thesis, Birkbeck College, University of London, non publié. 
MEAK, Lydia (1983). La selezione dell'informazione per l'interpretazione simultanea della literatura madica, monographie, Universita degli studi di Trieste, Scuola Superiore di Lingue Moderne per Interpreti e Traduttori, Trieste.

MOSER-MERCER, Barbara (1992). «Banking on Terminology Conference, Interpreters in the Electronics Age», Meta XXXVII(3), pp. 507-522.

NG, Bee Chin et Yasuko OBANA (1992). «The Significance of Speech Levels in English-Japanese Interpretation», The Interpreter's Newsletter, Special Issue 1, pp. 42-51.

OLERON, P. et H. NANPON (1964). «Recherches sur la traduction simultanée», Journal de Psychologie Normale et Pathologique 62, pp. 73-94

OVASKA, Paula (1987). Unfilled Pauses and Hesitations in Impromptu Speech and Simultaneous Interpretation. M.A. Thesis, University of Turku, non publié.

PETSCHE, H. (1993). «Hirnelektrische Vorgänge bei verbalem Denken», Neuropsychiatrie VII(1), pp. 13-17.

PINTER (KURZ), Ingrid (1969). Der Einfluss der Übung und Konzentration auf simultanes Sprechen und Hören. Thèse de doctorat, Université de Vienne, non publiée.

PÖCHHACKER, Franz (1993). «On the Science of Interpretation», The Interpreter's Newsletter 5, pp. 52-59.

PÖCHHACKER, Franz (1994). Simultandolmetschen als komplexes Handeln. «Language in Performance» 10, Tübingen, Narr.

SALEVSKY, Heidemarie (1992). «Dolmetschen - Objekt der Ubzersetzungsoder Dolmetschwissenschaft?» in Salevsky H., dir. (1992), Wissenschaftliche Grundlagen der Sprachmittlung. Berliner 
Beiträge zur Vbersetzungswissenschaft. Frankfurt am Main-BerlinBern-New York-Paris-Wien, Peter Lang, pp. 85-117.

SCHWEDA-NICHOLSON, Nancy (1986). "A United Nations Interpreter Survey: The Specialist/Generalist Controversy I», Multilingua $\mathrm{V}(2)$, pp. 67-80.

(1989). «An Interpreter Survey: The Specialist/ Generalist Controversy II», The Interpreter's Newsletter 2, pp. 38-45.

SELESKOVITCH, Danica (1968). L'Interprète dans les conférences internationales: problèmes de langage et de communication. Paris, Lettres modernes Minard.

modernes Minard.

(1975). Langage, langues et mémoire. Paris, Lettres

SHLESINGER, Miriam (1989). Simultaneous Interpretation as a Factor in Effecting Shifts in the Position of Texts on the Oral-Literate Continuum. M.A. Thesis, Faculty of Humanities, Tel Aviv University, non publié.

SNELL-HORNBY, Mary, Franz PÖCHHACKER et Klaus KAINDL, dir., (1993). Translation Studies: An Interdiscipline. Amsterdam/Philadelphia, John Benjamins.

STENZL, Catherine (1983). Simultaneous Interpretation Groundwork Towards a Comprehensive Model. M.A. thesis, Birkbeck College, University of London, non publié.

THIERY, Christopher (1975). Le Bilinguisme chez les interprètes de conférence professionnels. Thèse de Doctorat de $3^{e}$ cycle, Université Paris III, non publiée.

TIRKKONEN-CONDIT, Sonja, dir. (1991). Empirical Research in Translation and Intercultural Studies. Tübingen, Gunter Narr Verlag. 
TIRKKONEN-CONDIT, Sonja et John LAFFLING, dir. (1993). Recent Trends in Empirical Translation Research, Studies in Languages 28, University of Joensuu.

TOMMOLA, Jorma et Jukka HYÖNÄ (1990). «Mental Load in Listening, Speech Shadowing and Simultaneous Interpreting: a Pupillometric Studyn, in Tommola Jorma, dir. (1990), Foreign Language Comprehension and Production, AFinLA Yearbook, Turku.

TOMMOLA, Jorma et Pekka NIEMI (1986). «Mental Load in Simultaneous Interpreting: an On-line Pilot Studyy, in Evensen 1., dir. (1986), Nordic Research in Text Linguistics and Discourse Analysis.

TREISMAN, A. (1965). "The Effects of Redundancy and Familiarity on Translating and Repeating Back a Foreign and a Native Language», British Journal of Psychology 56, pp. 369-379.

VAN HOOF, Henri (1962). Théorie et pratique de l'interprétation. Munich, Max Hueber Verlag.

VIEZZI, Maurizio (1990). «Sight Translation, Simultaneous Interpretation and Information Retention», in Gran et Taylor, dir. (1990), pp. 54-60.

(1993). «Written Translation and Simultaneous Interpretation Compared and Contrasted: A Case Study», The Interpreter's Newsletter 5, pp. 94-100.

RÉSUMÉ: Évolution de la recherche empirique sur l'interprétation de conférence - Dans un historique de la recherche sur l'interprétation, les années 90 correspondent à une période de renouveau après une prise de conscience (années 50 et 60), une période expérimentale (années 65 à 75 ) et une période de stagnation due au protectionnisme de certains praticiens au pouvoir ( 75 à fin des années 80). Ce renouveau se traduit par une plus grande 
ouverture interdisciplinaire et inter-centres et par une sensibilisation à la recherche empirique, mais celle-ci reste faible: les chercheurs, surtout des interprètes-enseignants, sont peu nombreux et n'ont pas été formés à la recherche et aux disciplines cognitives pertinentes; les centres sont peu nombreux, petits, dispersés et sans infrastructure institutionnelle. Les perspectives d'évolution restent incertaines.

\section{ABSTRACT: Trends in Empirical Research into Conference} Interpreting - In the history of research on conference interpreting, the 1990s represent a period of revival. This follows a period of awareness (1950s and 1960s), an experimental period (1965-1975), and a period of stagnation due to the protectionist attitude of some influential practitioners in the field (1975 to the end of the 1980s). The revival is characterized by a more open interdisciplinary and inter-centre approach, as well as by a greater, although still moderate, interest in empirical research. Researchers, especially interpreter-instructors, are few and are not trained in research or in the relevant disciplines. Moreover, the centres are few, small, far apart and are lacking in institutional infrastructure. The future of research in the field thus remains uncertain. (Trans. by Paul Bandia) 